\title{
Black Hole Deconstruction
}

\author{
Frederik Denef*, Davide Gaiotto ${ }^{\dagger}$ \\ Andrew Strominger ${ }^{\dagger}$, Dieter Van den Bleeken* and Xi Yin ${ }^{\dagger}$
}

\begin{abstract}
A $D 4-D 0$ black hole can be deconstructed into a bound state of $D 0$ branes with a $D 6-\bar{D} 6$ pair containing worldvolume fluxes. The exact spacetime solution is known and resembles a $D 0$ accretion disk surrounding a $D 6-\bar{D} 6$ core. We find a scaling limit in which the disk and core drop inside an $A d S_{2}$ throat. Crossing this $A d S_{2}$ throat and the $D 0$ accretion disk into the core, we find a second scaling region describing the $D 6-\bar{D} 6$ pair. It is shown that the M-theory lift of this region is $A d S_{3} \times S^{2}$. Surprisingly, time translations in the far asymptotic region reduce to global, rather than Poincaré, time translations in this core $A d S_{3}$. We further find that the quantum mechanical ground state degeneracy reproduces the Bekenstein-Hawking entropy-area law.
\end{abstract}

* Institute for Theoretical Physics, K.U. Leuven, Celestijnenlaan 200D, B-3001 Leuven, Belgium

$\dagger$ Center for the Fundamental Laws of Nature, Jefferson Physical Laboratory, Harvard University, Cambridge, MA USA 


\section{Introduction}

A BPS black hole can be deconstructed in a variety of ways into zero-entropy, minimally charged bits - often wrapped branes. It is natural to suppose that black hole entropy is the ground state degeneracy of the quantum mechanics on the moduli space of such deconstructions. This quantum mechanics is expected to have a superconformal symmetry and provide the $C F T_{1}$ dual of the near-horizon $A d S_{2}$ geometry. The program to understand black holes in this way has never fully succeeded, although the hope that it may some day do so remains very much alive. Some reviews can be found in [1,2] and some recent progress in[3].

Prior attempts appear to have been missing a crucial ingredient brought to light in [4]. Up to now such analyses have mainly concerned like-charged (or mutually supersymmetric) black holes, which enjoy a no-force condition and can lie at any relative location. This leads to various volume divergences and singularities in the moduli space quantum mechanics. In [4] it was discovered that in the generic situation, when the black hole charge vectors point in different directions, there is a rich, but surprisingly tractable moduli space of solutions in which all constituents are bound. In this paper we will see that in some cases there is a scaling limit with a superconformal symmetry. This moduli space quantum mechanics is qualitatively different from the parallel charged case, and looks promising for an understanding of black hole microscopics.

In this paper we will consider the deconstruction of a single center charge $\left(P, q_{0}\right)$ $D 4-D 0$ black hole into a multicenter system consisting of a $D 6$ brane with flux $F=P / 2$ on the worldvolume, a $\bar{D} 6$ with flux $F=-P / 2$, and $\tilde{q}_{0}=q_{0}+\frac{P^{3}}{24} D 0$ branes, where $P \gg 1$. For simplicity we take the dimension of the D4 and D2 charge spaces to be one, but generalization is straightforward. This system appeared in [5] in the context of a derivation of the OSV conjecture, in which mainly the case $\tilde{q}_{0} \ll P^{3}$ was important. In this regime, a single centered black hole of charge $\left(P, q_{0}\right)$ does not exist, and the centers stay well separated from each other. However when $\tilde{q}_{0}$ becomes greater than $P^{3} / 12$, a phase transition occurs: the BPS configuration moduli space develops a branch in which the centers can approach each other arbitrarily close in coordinate distance, leading to a capped off $A d S_{2}$ throat of arbitrary depth. Such configurations are asymptotically indistinguishable from, and perhaps should be physically identified with, those of a single centered black hole. In this scaling limit there is a superconformal symmetry acting on the moduli space quantum mechanics, which one hopes is related to or identified with the $C F T_{1}$ dual of the the $A d S_{2}$ throat. 
In section 2 we review the full asymptotically flat $D 0-D 6-\bar{D} 6$ classical solution in terms of harmonic functions on $R^{3}$. In section 3 we write down the scaling solution, which is effectively obtained by simply dropping the constants in the harmonic functions (this is consistent only when certain charge constraints are satisfied). In the far region, the scaling solution looks like $A d S_{2} \times S^{2}$ and lifts to $A d S_{3} \times S^{2}$ in $M$-theory. In the interior there are pointlike $D 0$ brane singularities which are free to move as long as they stay on an "accretion disk" which lies on the plane equidistant to the $D 6$ and $\bar{D} 6$. In the central "near region", far inside the accretion disk, one finds a geometry whose $M$-lift is again $A d S_{3} \times S^{2}$, but now with global $A d S_{3}$. The $D 6$ and $\bar{D} 6$ are the north and south poles of the $S^{2}$. 1 In section 4 we analyze the (super)symmetries of the solution, and find a nontrivial relation between the generators of the near and far $A d S_{3}$ s. In particular the twisted global time generator $\bar{L}_{0}^{\text {near }}-J^{3 \text { near }}$ of the near region is the Poincare time generator of the far $A d S_{3}$ as well as the Hamiltonian of the full asymptotically flat geometry. This surprising fact may provide a derivation of the conjecture $[7,8]$ that the black hole entropy is the degeneracy of quantum eigenstates with respect to the global time generator of the near horizon $A d S_{2}$. In section 5 we analyze supersymmetric branes which wrap the $S^{2}$ "horizon" and correspond to D0 branes blown up via the Myers effect. In section 6, following earlier work [3], we show that such wrapped branes have a huge degeneracy, and can account for the Bekenstein-Hawking entropy. This is a positive indication that the superconformal quantum mechanics of the deconstruction given here may give a good picture of the $D 4-D 0$ quantum black hole.

\section{The full classical solution}

In this section we present the asymptotically flat four-dimensional classical solution corresponding to $\tilde{q}_{0} D 0$-branes and a $D 6-\bar{D} 6$ pair with fluxes inducing $D 4-D 2-D 0$ charges $\left(\frac{P}{2}, \pm \frac{P^{2}}{8},-\frac{P^{3}}{48}\right)$, as well as its five-dimensinal M-theory lift. This is a special case of a more general class of solutions described in [9]. For notational simplicity we consider the case of a single vector multiplet (two $U(1) \mathrm{s}$ ) but the results are easily generalized.

1 This lift of $D 6-\bar{D} 6$ to $A d S_{3} \times S^{2}$ also relates the derivations of OSV given in [5], where the two factors of $Z_{\text {top }}$ come from a $D 6$ and a $\bar{D} 6$, to that of [6] where they come from the north and south poles of an $S^{2}$. 
The solution is constructed from the four harmonic functions on $R^{3}$

$$
\begin{aligned}
& H^{0}=h^{0}+\frac{1}{\left|x-x_{D 6}\right|}-\frac{1}{\left|x-x_{\bar{D} 6}\right|}, \\
& H^{1}=h^{1}+\frac{P}{2} \frac{1}{\left|x-x_{D 6}\right|}+\frac{P}{2} \frac{1}{\left|x-x_{\bar{D} 6}\right|}, \\
& H_{1}=h_{1}-\frac{P^{2}}{8} \frac{1}{\left|x-x_{D 6}\right|}+\frac{P^{2}}{8} \frac{1}{\left|x-x_{\bar{D} 6}\right|}, \\
& H_{0}=h_{0}-\frac{P^{3}}{48} \frac{1}{\left|x-x_{D 6}\right|}-\frac{P^{3}}{48} \frac{1}{\left|x-x_{\bar{D} 6}\right|}+\sum_{i=1}^{\tilde{q}_{0}} \frac{1}{\left|x-x_{D 0}^{i}\right|},
\end{aligned}
$$

in a self-evident notation. An integrability condition (the existence of $\omega$ below) restricts the brane positions to obey the constraints

$$
\begin{gathered}
h^{0}+\frac{1}{\left|x_{D 6}-x_{D 0}^{i}\right|}=\frac{1}{\left|x_{\bar{D} 6}-x_{D 0}^{i}\right|}, \\
\left(h_{0}-\frac{P}{2} h_{1}-\frac{P^{2}}{8} h^{1}+\frac{P^{3}}{48} h^{0}\right)+\sum_{i=1}^{\tilde{q}_{0}} \frac{1}{\left|x_{D 6}-x_{D 0}^{i}\right|}=\frac{P^{3}}{6\left|x_{\bar{D} 6}-x_{D 6}\right|} .
\end{gathered}
$$

From the harmonic functions (2.1) we form the algebraic combinations

$$
\begin{gathered}
Q=H_{1}+\frac{\left(H^{1}\right)^{2}}{2 H^{0}}, \\
J=-\frac{H_{0}}{2}+\frac{\left(H^{1}\right)^{3}}{6\left(H^{0}\right)^{2}}+\frac{H^{1} H_{1}}{2 H^{0}},
\end{gathered}
$$

and

$$
S=2 \pi \sqrt{\frac{2}{9} H^{0} Q^{3}-\left(H^{0} J\right)^{2}} .
$$

In terms of these quantities the complex Calabi-Yau modulus is

$$
z=\frac{\pi H^{1}+i \frac{\partial S}{\partial H_{1}}}{\pi H^{0}+i \frac{\partial S}{\partial H_{0}}}
$$

The $4 \mathrm{D}$ metric is

$$
d s_{4}^{2}=-\frac{\pi}{S}(d t+\omega)^{2}+\frac{S}{\pi} d \vec{x}^{2},
$$

$\omega$ here is the 1-form that satisfies

$$
*^{3} d \omega=H_{0} d H^{0}+H_{1} d H^{1}-H^{0} d H_{0}-H^{1} d H_{1} .
$$

The gauge fields strengths are

$$
d A^{\Lambda}=d\left(S^{-1} \frac{\partial S}{\partial H_{\Lambda}}(d t+\omega)\right)+*^{3} d H^{\Lambda},
$$

for $\Lambda=0,1$.

The M-theory lift is given by

$$
d s^{2}=-\frac{9}{8} Q^{-2}\left(d t+\omega+2 J\left(d x^{5}+\omega^{0}\right)\right)^{2}+Q\left(H^{0} d \vec{x}^{2}+\left(H^{0}\right)^{-1}\left(d x^{5}+\omega^{0}\right)^{2}\right),
$$

where $\omega^{0}$ is determined by $d \omega^{0}=*^{3} d H^{0}$. 


\section{The scaling solutions}

In this section we describe the "far-horizon" and "near-horizon" scaling regions, both of which M-lift to (quotients of) $A d S_{3} \times S^{2}$.

We are looking for "scaling solutions" obtained by rescaling all coordinates $x \rightarrow \lambda \tilde{x}$ and taking $\lambda \rightarrow 0$ while keeping $\tilde{x}$ finite. This effectively amounts to dropping the constant terms $h$ in the harmonic functions (2.1). The charges are then sitting at different locations far down a very long throat.

In this scaling limit, the integrability conditions (2.2) at the $D 0$ location force the $D 0$-branes to be equidistant from the $D 6$ and the $\bar{D} 6$. Hence all $\tilde{q}_{0}$ of them lie in a plane, say $x^{3}=0$. resembling an accretion disk or ring surrounding the $D 6-\bar{D} 6$ pair. The integrability condition (2.3) at the $D 6$ location fixes the distance $R_{6}$ between the $D 6$ and this plane. Hence the moduli space of solutions is the $2 \tilde{q}_{0}$ positions of the $D 0 \mathrm{~s}$ on $R^{2}$.

Since the distance between any D0 and the D6 is at least $R_{6}$, (2.3) implies $\tilde{q}_{0} \geq P^{3} / 12$, so we only have scaling solutions when this constraint on the charges is met. We are in particular interested in the regime

$$
\tilde{q}_{0}>>P^{3} / 12 \text {. }
$$

In this case generically the $D 6$ and $\bar{D} 6$ will be much closer to the plane than to the $D 0$ s. For example if the $D 0 \mathrm{~s}$ all lie on a ring of radius $R_{0}$ the distance $R_{6}$ between the $D 6$ and the plane satisfies

$$
\frac{P^{3}}{12} \frac{1}{R_{6}}=\frac{\tilde{q}_{0}}{\sqrt{R_{0}^{2}+R_{6}^{2}}}
$$

and $R_{0}>>R_{6}$. While the full geometry is pretty complicated, it simplifies in the far region $|x|>>R_{0}$ and near region $|x|<<R_{0}$.

\subsection{Far region}

Far outside the $D 0$ ring, the fine structure of the pointlike branes disappears down the throat and the geometry following from (2.1) with $h=0$ is simply the near horizon geometry of a $D 4-D 0$ black hole. The M-theory lift is

$$
\frac{\left(24 \tilde{q}_{0}-P^{3}\right)}{8 P} d x_{5}^{2}+\frac{3 r}{P} d t d x_{5}+\frac{P^{2}}{2 r^{2}} d r^{2}+\frac{P^{2}}{2}\left(d \eta^{2}+\sin ^{2} \eta d \psi^{2}\right)
$$


where we have traded $\vec{x}$ for $r, \eta, \psi$. The periodic identification $x^{5} \sim x^{5}+4 \pi$ makes this an $S L(2, R)_{L}$ quotient of $A d S_{3} \times S^{2}$. On the boundary $r \rightarrow \infty$, the conformal metric is simply

$$
d s_{2}^{2}=d t d x^{5}
$$

where $S L(2, R)_{L}\left(S L(2, R)_{R}\right)$ acts in the standard fashion on the left (right) null coordinate $x^{5}(t)$. In particular we see that the Hamiltonian $\partial_{t}$ of the original asymptotically flat region generates null translations of the unbroken $S L(2, R)_{R}$.

$A d S_{3}$ quotients of this type were analyzed in [10]. While $t$ is a standard null coordinate on the Poincare diamond of the boundary of $A d S_{3}, x^{5}$ in fact turns out to be a Rindler coordinate. The $S L(2, R)_{L}$-invariant $A d S_{3}$ vacuum then gives, after $x^{5}$ identifications, a thermal state for the left movers of the boundary CFT. The entropy of this thermal state agrees with that of the $D 4-D 0$ black hole.

\subsection{Near region}

Now we consider the near region, far inside the accretion disk. It is convenient to work in prolate spheroidal coordinates $\rho, \eta, \phi$ (plus $x^{5}$ and $\left.t\right)$ :

$$
\begin{aligned}
& \left|x-x_{D 6}\right|=R_{6}(\cosh \rho+\cos \eta), \\
& \left|x-x_{\bar{D} 6}\right|=R_{6}(\cosh \rho-\cos \eta) .
\end{aligned}
$$

Lines of constant $\rho$ are ellipsoids with foci at the $D 6$ and $\bar{D} 6$ locations, while $\eta$ is an angle around the ellipsoid. In this coordinate system, the functions appearing in the metric are

$$
\begin{gathered}
Q=-\frac{P^{2}}{4 R_{6} \cos \eta} \\
J=\frac{P^{3}}{24 R_{6}} \frac{\cosh \rho}{\cos ^{2} \eta}-\frac{1}{2} \sum_{i=1}^{\tilde{q}_{0}} \frac{1}{\left|x-x_{D 0}^{i}\right|} .
\end{gathered}
$$

The near region is characterized by

$$
|x|<<\left|x_{D 0}^{i}\right| \sim R_{0}
$$

This allows us to replace the summation in the rhs of (3.7) by a constant. This constant is fixed by the the integrability condition (2.3). One then has

$$
J=\frac{P^{3}}{24 R_{6}}\left(\frac{\cosh \rho}{\cos ^{2} \eta}-1\right) .
$$


The last term in $H_{0}$ reduces to the constant $\frac{P^{3}}{12 R_{6}}$, and $\omega$ becomes

$$
\omega=\frac{P^{3}}{3 R_{6}} \frac{\sinh ^{2}\left(\frac{\rho}{2}\right) \sin ^{2} \eta d \phi}{\left(\cosh ^{2} \rho-\cos ^{2} \eta\right)} .
$$

After a linear change of coordinates

$$
\begin{aligned}
t & =\frac{P^{3}}{3 R_{6}} \tau, \\
x_{5} & =2(\tau+\theta), \\
\phi & =\psi+\tau-\theta,
\end{aligned}
$$

the metric (2.11) takes the global $A d S_{3} \times S^{2}$ form

$$
\frac{P^{2}}{2}\left(d \eta^{2}+\sin ^{2} \eta d \psi^{2}\right)+2 P^{2}\left(-\cosh ^{2}\left(\frac{\rho}{2}\right) d \tau^{2}+\sinh ^{2} \frac{\rho}{2} d \theta^{2}+\frac{1}{4} d \rho^{2}\right)
$$

with no identifications except the standard $2 \pi$ identifications of $\psi$ and $\theta$. This near region metric has an enhanced $S L(2, R)_{L} \times S U(1,1 \mid 2)$ superisometry group.

\section{Supersymmetry}

Calabi-Yau compactification of Type IIA or M theory preserves eight supersymmetries. Of these, the general multicenter solution preserves four which we denote

$$
{ }^{ \pm} Q^{ \pm}
$$

where the left (right) superscript denotes the charges under shifts of $x^{5}(\phi)$. Commutators of these preserved charges give the Hamiltonian $\left(i \partial_{t}\right)$

$$
\left\{{ }^{A} Q^{\alpha},{ }^{B} Q^{\beta}\right\}=\epsilon^{A B} \epsilon^{\alpha \beta} H+\text { central terms, }
$$

where $\alpha, A= \pm$. The supercharges commute with $H$ :

$$
\left.{ }^{ \pm} Q^{ \pm}, H\right]=0
$$

In the near and far $A d S$ regions (3.3) and (3.12), the four supercharges are locally enhanced to eight $S U(1,1 \mid 2)$ superconformal symmetries. We wish to understand which four of the eight supersymmetries in each of the near and far regions are globally preserved. 


\subsection{Near region}

Let us denote the eight supercharges of the near region in a standard convention

$$
{ }^{L_{0}} G_{\bar{L}_{0}}^{J^{3}}={ }^{ \pm} G_{ \pm \frac{1}{2}}^{ \pm}
$$

The $G$ s have eigenvalues $\pm \frac{1}{2}$ under the action of

$$
\begin{aligned}
& \bar{L}_{0}^{\text {near }}=\frac{1}{2}\left(\partial_{\tau}-\partial_{\theta}\right), \\
& L_{0}^{\text {near }}=\frac{1}{2}\left(\partial_{\tau}+\partial_{\theta}\right), \\
& J_{\text {near }}^{3}=\partial_{\psi},
\end{aligned}
$$

Using the coordinate transformation (3.11) we find

$$
\frac{P^{3}}{3 R_{6}} H=2\left(\bar{L}_{0}^{\text {near }}-J_{\text {near }}^{3}\right) .
$$

The four linear combinations of the eight ${ }^{ \pm} G_{ \pm \frac{1}{2}}^{ \pm}$which correspond to ${ }^{ \pm} Q^{ \pm}$can then be deduced by demanding that they commute with $\bar{L}_{0}^{\text {near }}-J_{\text {near }}^{3}$ as in (4.3). One finds

$$
{ }^{ \pm} Q^{+}={ }^{ \pm} G_{\frac{1}{2}}^{+}, \quad{ }^{ \pm} Q^{-}={ }^{ \pm} G_{-\frac{1}{2}}^{-} .
$$

\subsection{Far Region}

In the far region we similarly denote the supercharges by

$$
{ }^{L_{0}} F_{\bar{L}_{0}}^{J^{3}}={ }^{ \pm} F_{ \pm \frac{1}{2}}^{ \pm}
$$

These have eigenvalues $\pm \frac{1}{2}$ under the action of

$$
\begin{aligned}
L_{0}^{\mathrm{far}} & =2 \partial_{5}, \\
J_{\text {far }}^{3} & =\partial_{\phi} .
\end{aligned}
$$

Employing the transformation to global coordinates for the $A d S_{3}$ one finds

$$
\frac{P^{3}}{3 a} H=\bar{L}_{0}^{\mathrm{far}}-\frac{1}{2}\left(\bar{L}_{1}^{\mathrm{far}}+\bar{L}_{-1}^{\mathrm{far}}\right) .
$$

It then follows using (4.3) that

$$
{ }^{ \pm} Q^{ \pm}={ }^{ \pm} F_{\frac{1}{2}}^{ \pm}-{ }^{ \pm} F_{-\frac{1}{2}}^{ \pm} .
$$


Comparing (4.9) and (4.5) with the help of the coordinate transformations (3.11) one finds

$$
\begin{aligned}
2 L_{0}^{\text {near }} & =\bar{L}_{0}^{\text {far }}-\frac{1}{2}\left(\bar{L}_{1}^{\mathrm{far}}+\bar{L}_{-1}^{\mathrm{far}}\right)+2 L_{0}^{\mathrm{far}}, \\
\bar{L}_{0}^{\text {near }}-L_{0}^{\text {near }} & =J^{3 \mathrm{far}}-L_{0}^{\mathrm{far}}, \\
J_{\text {near }}^{3} & =J_{\text {far }}^{3} .
\end{aligned}
$$

\section{Wrapped branes}

In this section we describe some supersymmetric wrapped branes, whose microstates will be argued in the next section to dominate the entropy for large D0-charges.

\subsection{Probe supersymmetries}

Consider an M2 brane wrapped on the $S^{2}$ in the near geometry, and sitting at the center $\rho=0$ of the $A d S_{3}$. As shown in [11] this breaks half the supersymmetry. Clearly this leaves unbroken both $S U(2)$ rotations of the $S^{2}$ and $U(1)$ rotations of the $A d S_{3}$. It follows that the unbroken supercharges of the near region must be

$$
{ }^{+} G_{\frac{1}{2}}^{ \pm}, \quad-G_{-\frac{1}{2}}^{ \pm} .
$$

Comparing with (4.7) we see that there are two globally unbroken supercharges

$$
{ }^{+} Q^{+}, \quad{ }^{-} Q^{-}
$$

The existence of two unbroken global supercharges is a generic feature of a supersymmetric probe configuration in backgrounds with four supercharges. Configurations preserving the same supersymmetries also exist which carry angular momentum in the $A d S_{3}$ [11]. These are given by $\rho=\rho_{0}, x^{5}=x_{0}^{5}$, i.e. $\theta=-\tau+\frac{x_{0}^{5}}{2}$.

\subsection{Explicit solutions}

A supersymmetric probe solution exists for M2 branes in the full geometry. The Killing spinors of the metric (2.11) are given in terms of the covariantly constant spinors of the $4 d$ hyper-Kähler base space [12]

$$
d s^{2}=H^{0} d \vec{x}^{2}+\left(H^{0}\right)^{-1}\left(d x^{5}+\omega^{0}\right)^{2} .
$$


The kappa-symmetry constraint for M2 branes static with respect to the time $t$ requires the M2 brane to wrap a supersymmetric cycle in the $4 d$ base space, i.e. a cycle holomorphic for one of the complex structures of the base space.

The geometry of the base space only depends on $H^{0}$ and is the same for the full geometry and for the $A d S_{3} \times S^{2}$ near geometry. This is true even for the asymptotically flat geometry, as long the constant terms in the harmonic functions satisfy $h^{0}=0$. The natural choice for the moduli at infinity, i.e. the attractor moduli for the $D 4-D 0$ black hole, satisfy this condition. A non-zero $h^{0}$ would require only a minor modification of this construction. Hence the same equation $\rho=\rho_{0}, x^{5}=x_{0}^{5}$ that defines a supersymmetric $M 2$ brane that wraps the sphere in $A d S_{3} \times S^{2}$ also defines a supersymmetric M2 brane in the full geometry. This fact can be confirmed by looking at the complex structures of the base space. A natural set of complex coordinates is given by

$$
U=e^{-i \frac{x^{5}}{2}} \tanh \frac{\rho}{2}, \quad V=e^{i \phi} \sinh \rho \sin \eta
$$

The M2 brane probe sits at $U=U_{0}$. Those are the only compact holomorphic cycles in the geometry.

In the near geometry $(3.12)$, these probes move on a circle of constant radius: $\rho=\rho_{0}$, $\theta=\frac{x_{0}}{2}-\tau$, and have angular momentum

$$
J_{\theta}=-\frac{1}{4 \pi} \frac{P^{3}}{3} \sinh ^{2} \frac{\rho}{2}
$$

Reduced to four dimensions, the M2 branes become ellipsoidal D2 branes, with the D6 and $\bar{D} 6$ at the foci of the ellipsoid, and worldvolume flux

$$
F=-\frac{J_{\theta}}{2} \sin \eta d \eta \wedge(d \phi-2 d \tau)-\frac{1}{8 \pi} \frac{P^{3}}{3} \sin \eta d \eta \wedge d \tau
$$

The flux induces a $D 0$ charge $q_{0}=-J_{\theta}$, as expected from the identification of charges between the near geometry and the asymptotic geometry. This configuration is reminiscent of a supertube [13], but unlike the supertube, here both the fundamental string charge density and, proportional to this, the four dimensional $J_{\phi}$ angular momentum density are zero; the contributions from the Born-Infeld and Chern-Simons parts of the D2 Lagrangian cancel. Indeed, the M2 at $x_{5}=x_{5}^{0}$ is transversal to the M-theory circle, and does not carry any angular momentum $J_{\psi}\left(=J_{\phi}\right)$ along the $S^{2}$ it wraps. This is in contrast to the D0 particle probes, which do carry $J_{\phi}$ angular momentum in the $D 6-\bar{D} 6$ background. Finally, 
note that as in [3] these ellipsoidal D2 branes move in the Calabi-Yau as particles in a magnetic field proportional to $P$, because of the background $F^{(4)}$ RR-field.

This analysis shows the existence of a new, larger moduli space of multicenter configurations carrying $D 4-D 0$ charges: the moduli space of a system formed by the $D 6-\bar{D} 6$ pair, a certain number of $D 0$ branes and several ellipsoidal D2 branes carrying the extra D0 charge.

\section{Black hole entropy}

In this section we sketch how the Bekenstein-Hawking entropy emerges from the counting of microstates for a system that includes the ellipsoidal D2s.

The fastest way to understand the state-counting for this system is to reduce it to a problem previously analyzed in [3]. In that work, the superconformal chiral primary bound states of $D 0$ branes in the near-horizon $A d S_{2} \times S^{2}$ geometry of a $D 4$ - $D 0$ black hole were counted. A large degeneracy arises from non-abelian $D 0$ configurations which puff up via the Myers effect and form a $D 2-D 0$ bound state wrapping the horizon. This carries no $D 2$ charge as measured at infinity. Such a D2-D0 configuration couples to the background $F^{(4)} \mathrm{RR}$ field and hence sees an effective magnetic field on the Calabi-Yau. Hence the supersymmetric ground states are highly degenerate lowest Landau levels. Partitioning the $D 0$ charge among such configurations produces an exponential growth of states which exactly matches the Bekenstein-Hawking entropy.

If we lift the calculation of [3] to M-theory, the near horizon geometry becomes $A d S_{3} \times$ $S^{2}$, the horizon-wrapped $D 2$ becomes a horizon wrapped $M 2$, and the $D 0$ charge becomes orbital angular momentum. Hence in M-theory the supersymmetric states of a horizon wrapped $M 2$ are highly degenerate lowest Landau levels on the Calabi-Yau and reproduce the $D 4-D 0$ entropy.

In the current paper, we have deconstructed the $D 4-D 0$ black hole into zero-entropy $D 0-D 6-\bar{D} 6$ bits, which have a classical moduli space. It is natural to try to identify the black hole microstates with the supersymmetric ground states of the moduli space quantum mechanics (including the non-abelian interactions of near-coincident $D 0$ s which are crucial for the Myers effect)). In the $A d S_{3} \times S^{2}$ centered between the $D 6-\bar{D} 6$ pair, the $D 0$-branes have all been taken off to infinity. The entropy should appear as the number of ways of reincorporating these $D 0 \mathrm{~s}$ in this $A d S_{3} \times S^{2}$. Using the wrapped $M 2$ this counting proceeds exactly as above and gives the desired result. 
In addition to reproducing all the successes of [3], the current analysis potentially has several important advantages. The main observation of [3] was that the horizonwrapped bound states could account for the entropy. Reference [3] did not attempt either to provide a systematic framework in which higher order corrections to the entropy could be computed, or to derive or justify the conjecture of [7,8] that states should be counted in the global, rather than the Poincare, time of the near horizon $A d S_{2}$. Perhaps these shortcomings can both be overcome in the context of the deconstructed black hole quantum mechanics discussed here.

\section{Acknowledgements}

We would like to thank Miranda Cheng, Greg Moore and Toine Van Proeyen for discussions. This work was supported in part by DE-FGO2-91ER40654 and the Center for the Fundamental Laws of Nature at Harvard University, and by the Belgian Federal Office for Scientific, Technical and Cultural Affairs through the "Interuniversity Attraction Poles Programme - Belgian Science Policy" P5/27 and by the European Community's Human Potential Programme under contract MRTN-CT-2004-005104 "Constituents, fundamental forces and symmetries of the universe". DVdB is supported by an Aspirant scholarship from the FWO - Vlaanderen. XY is supported by a Junior Fellowship from the Harvard Society of Fellows. 


\section{References}

[1] R. Britto-Pacumio, J. Michelson, A. Strominger and A. Volovich, 'Lectures on superconformal quantum mechanics and multi-black hole moduli spaces," proceedings of the Akuyreri, Iceland summer school on string theory August, 1999, arXiv:hepth/9911066.

[2] S. D. Mathur, "The fuzzball proposal for black holes: An elementary review," Fortsch. Phys. 53, 793 (2005) arXiv:hep-th/0502050.

[3] D. Gaiotto, A. Strominger and X. Yin, "Superconformal black hole quantum mechanics," JHEP 0511, 017 (2005) arXiv:hep-th/0412322.

[4] F. Denef, "Supergravity flows and D-brane stability," JHEP 0008, 050 (2000) arXiv:hep-th/0005049.

[5] F. Denef and G. W. Moore, "Split states, entropy enigmas, holes and halos," arXiv:hep-th/0702146.

[6] D. Gaiotto, A. Strominger and X. Yin, "From AdS(3)/CFT(2) to black holes / topological strings," arXiv:hep-th/0602046.

[7] G. W. Gibbons and P. K. Townsend, "Black holes and Calogero models," Phys. Lett. B 454, 187 (1999) arXiv:hep-th/9812034.

[8] R. Britto-Pacumio, A. Strominger and A. Volovich, "Two-black-hole bound states," JHEP 0103, 050 (2001) arXiv:hep-th/0004017.

[9] B. Bates and F. Denef, "Exact solutions for supersymmetric stationary black hole composites," arXiv:hep-th/0304094.

[10] J. M. Maldacena and A. Strominger, "AdS(3) black holes and a stringy exclusion principle," JHEP 9812, 005 (1998) arXiv:hep-th/9804085.

[11] A. Simons, A. Strominger, D. M. Thompson and X. Yin, "Supersymmetric branes in $\operatorname{AdS}(2) \times \mathrm{S}^{* *} 2 \times \mathrm{CY}(3), "$ arXiv:hep-th/0406121.

[12] J. P. Gauntlett, J. B. Gutowski, C. M. Hull, S. Pakis and H. S. Reall, "All supersymmetric solutions of minimal supergravity in five dimensions," Class. Quant. Grav. 20, 4587 (2003) arXiv:hep-th/0209114.

[13] D. Mateos and P. K. Townsend, "Supertubes," Phys. Rev. Lett. 87, 011602 (2001) arXiv:hep-th/0103030. 